\begin{tabular}{l|l} 
REVISTA & $\begin{array}{l}\text { Revista Educación } \\
\text { ISSN: 0379-7082 } \\
\text { ISSN: 2215-2644 } \\
\text { revedu@ gmail.com } \\
\text { Universidad de Costa Rica } \\
\text { Costa Rica }\end{array}$
\end{tabular}

\title{
Mediación tecnológica de la enseñanza: Entre artefactos, modelos y rol docente
}

\author{
Ligarretto Feo, Rennier Estefan \\ Mediación tecnológica de la enseñanza: Entre artefactos, modelos y rol docente \\ Revista Educación, vol. 45, núm. 2, 2021 \\ Universidad de Costa Rica, Costa Rica \\ Disponible en: https://www.redalyc.org/articulo.oa?id=44066178019 \\ DOI: https://doi.org/10.15517/revedu.v45i1.42999
}

\section{(c) 1 (1)}

Esta obra está bajo una Licencia Creative Commons Atribución-NoComercial-SinDerivar 3.0 Internacional. 


\title{
Mediación tecnológica de la enseñanza: Entre artefactos, modelos y rol docente
}

\author{
Technology Mediation in Teaching: Artifacts, Models and the Role of Teachers \\ Rennier Estefan Ligarretto Feo \\ Pontificia Universidad Javeriana, Colombia \\ DOI: https://doi.org/10.15517/revedu.v45i1.42999 \\ Redalyc: https://www.redalyc.org/articulo.oa? \\ rennierligarretto@javeriana.edu.co \\ $\mathrm{id}=44066178019$
}

iD https://orcid.org/0000-0002-8666-8284

Recepción: 03 Agosto 2020

Aprobación: 21 Octubre 2020

\section{Resumen:}

Este ensayo se ocupa de presentar una reflexión para comprender la noción de mediación tecnológica de la enseñanza. Para ello, resulta necesario analizar las implicaciones pedagógicas del uso de la tecnología como mecanismo instrumental o artefacto cultural para la mediación, este objeto de discusión centra la mirada en dimensiones políticas y educativas propias de una institución social. La escuela de la sociedad de la información y del conocimiento requiere reflexionar sobre el rol docente, los modelos teóricos, las estrategias didácticas y narrativas digitales que permiten flexibilizar la práctica educativa y significar el uso de la tecnología como una mediación cultural de la enseñanza. Las consideraciones finales resaltan la importancia de incorporar principios conectivistas para la mediación tecnológica de la enseñanza, tales como el reconocimiento de la tecnología como artefacto cultural permeado por dimensiones económicas, políticas y sociales necesarias de analizar desde el quehacer de las personas docentes.

Palabras Clave: Tecnologías de Información y Comunicación, TIC, Mediación, Cultura digital, Práctica docente, Conectivismo.

\section{Abstract:}

This study reflects on understanding the notion of technological mediation as a teaching tool by, first, analyzing the pedagogical undertones regarding the use of technology as an instrumental mechanism or cultural artifact for mediation. This subject of discussion focuses on the political and educational aspects of society. Education as part of an information and knowledgebased society must contemplate the role of teachers, theoretical models, didactic strategies and digital narratives to streamline education and incorporate technology as a cultural mediation tool for teaching. Final considerations highlight the importance of incorporating connectivism principles for the technological mediation in teaching. This involves acknowledging technology as a cultural artifact permeated by an economic, political and social dimension and analyzed from a teaching perspective.

KeYwords: Information and Communication Technologies, ICT, Mediation, Digital Culture, Teaching Practice, Connectivism.

\section{INTRODUCCIÓN}

La tecnología como producto humano epocal refleja los usos, prácticas y significados sociales que permean a la sociedad. La escuela como institución social no ha sido ajena a este proceso de transformación pues las resistencias frente a la apropiación educativa de la tecnología se presentan desde los inicios de los medios de comunicación masivos, como la radio y la televisión, hasta las fronteras digitales de la incorporación de inteligencia artificial con la intención de medir fenómenos cognitivos propios del proceso de enseñanza y aprendizaje. Un primer paso para comprender la noción de mediación tecnológica en la enseñanza implica observarla desde la dimensión física, es decir como una cosa, como un objeto material, preguntándonos su significado en relación con el uso de los dispositivos hasta las relaciones de poder constructo de una mirada de progreso de las ciencias de la educación.

Internet como tecnología de la sociedad de la información y del conocimiento, niega la geografía (Cairncross, 1997), y expande las formas comunicativas a narrativas digitales propias de una generación que trasciende las fronteras análogas y digitales. La mediación como fenómeno edu-comunicativo establece 
nuevas prácticas en torno a la acción educativa, las y los maestros deben fomentar el uso de la tecnología desde modelos teóricos, que conciban el aprendizaje en red; una mediación cultural implica generar prácticas reflexivas alrededor del saber, pensar y conocer en la relación activa del estudiante-profesor.

Finalmente, reflexionar sobre otras mediaciones de la tecnología para la enseñanza permite desprender una lógica instrumental que ha centrado la mirada en reducir brechas digitales de acceso, pero que desconoce una dimensión cultural de la mediación tecnológica que permite transformar roles, artefactos, ambientes de aprendizaje a narrativas propias de una cultura digital.

A partir de lo anterior, el texto expone las formas instrumentales y culturales de mediar los procesos de enseñanza y aprendizaje con tecnología. Para ello, se confirma la relación histórica entre la tecnología y los procesos de cambio social, al indicar cómo discursos de progreso y modernidad reflejan una dimensión política y económica de incorporar tecnología en la escuela. Seguido, se aborda la noción de mediación tecnológica para reflejar una tendencia a instrumentalizar la práctica docente, manteniendo concepciones educativas tradicionales sobre el aprendizaje y que, por el contrario, una concepción profunda de la mediación implica flexibilizar el rol, modelo y didácticas acordes a una generación digital.

Estos y otros elementos permiten establecer nuevas formas para analizar la mediación tecnológica de la enseñanza en un sistema educativo que resiste apropiar tecnología.

\title{
Referentes conceptuales: Tecnología: De la Herramienta AL ARTEFACTo CULTURAL
}

\author{
¡Ciegas y extrañamente sigilosas! \\ Durarán más allá de nuestro olvido; \\ no sabrán nunca que nos hemos ido
}

El poema de Borges (1996) titulado Las Cosas trae a la memoria los elementos materiales que nos acompañan y simbolizan en vida; estos sobreviven al paso del tiempo, pues representan las cargas simbólicas presentes en la interacción con el otro, al tiempo que constituyen un producto cultural propio de nuestra sociedad.

Al observar las cosas y su naturaleza en la sociedad, es posible centrar la mirada en la tecnología como producto de la cultura que ha transformado a la sociedad. Desde una mirada antropológica es posible pensar que la relación humana con las cosas comparta una historia común y que dichas cosas deben ser interpretadas a partir del modelado y conexión que le otorgan los individuos. En consecuencia, las relaciones simbólicas o materiales siempre están presentes en los objetos, la Techne como proceso de transformación y réplica natural que incluye a la tecnología como expresión propia de la transformación social (Esposito, 2016).

En este sentido, los artefactos deben ser entendidos como productos materiales y simbólicos de la sociedad que, en su expresión mecánica, industrial o digital, están presentes en las dimensiones sociales, culturales, artísticas, educativas y políticas. Sobre esta última dimensión, la historia muestra cómo las cualidades políticas derivadas de los usos sociales del artefacto tecnológico han sido clave para generar una cultura del progreso social. En palabras de Winner (1985):

El cambio tecnológico conlleva una amplísima muestra de motivos humanos, de los cuales el deseo de obtener dominio sobre los demás no es el menos frecuente, incluso aunque ello implique un sacrificio ocasional respecto a los costes y cierta violencia en los modos de conseguir más a partir de menos (p. 3).

$\mathrm{Al}$ detenernos sobre esta afirmación, se reconoce que los usos de los artefactos operan a partir de dispositivos de poder, la noción de dispositivo disciplinario como un ejercicio de control presente en acciones como la vigilancia jerárquica, la sanción normalizadora y el examen (Foucault,1968); estos mecanismos están presentes en la educación como un sistema para la regulación del proceso de enseñanza y aprendizaje. 
En este proceso, los desarrollos tecnológicos presentan una relación simbiótica con la cultura al estar presentes en todas las dimensiones de la sociedad. Si bien su expresión resulta más evidente en dimensiones políticas, también se hace necesario analizar su incidencia en la educación como institución social fundada para conservar la cultura y prepararla para convivir en sociedad. Para ello, es preciso iniciar desde la relación presente entre la ciencia, la tecnología y la sociedad como partes constitutivas de la cultura occidental. La Organización de Estados Iberoamericanos para la educación, la ciencia y la cultura (2001) indicó que:

La concepción clásica de las relaciones entre la ciencia y la tecnología con la sociedad, es una concepción esencialista y triunfalista, que puede resumirse en la simple ecuación, el llamado modelo lineal de desarrollo: +ciencia = +tecnología $=$ + riqueza $=+$ bienestar $\operatorname{social}($ p. 2).

En este contexto se presenta de manera clara cómo los usos de la ciencia y la tecnología marcan los lineamientos para el progreso de la sociedad, la industrialización y las posteriores transformaciones propias de la era de la información, lo que nos permite reconocer la incidencia de la tecnología en la sociedad (Castells, 1999).

En complemento, la noción de modernidad como fenómeno social, económico y político ha atravesado los procesos de globalización, los cuales han sido base para la constitución de las naciones cada vez mayormente interconectadas. Sobre la nación como concepto; los postulados de Smith (1983) reconocen a esta misma como una forma moderna de soberanía que permitió el nacimiento de la ciudadanía como escenario para ejercer derechos y deberes propios que nos diferenciaban de los otros (Elbaz y Helly,1996). Los discursos propios de la nación y ciudadanía han sido utilizados como herramientas para generar cohesión social. En este proceso, el lenguaje y la pedagogía se han constituido como estrategias para estructurar las funciones de la educación en la sociedad moderna.

Con los posteriores desarrollos tecnológicos, en particular la aparición de internet sobre la década de los noventa, las dimensiones sociales, económicas, culturales, artísticas y educativas se han transformado. Esto obedece a varios aspectos: el primero es que internet, a diferencia de otra tecnología de época, ha evolucionado de una manera rápida y progresiva, la ley de Moore (1975) planteó en su momento que entre 2 a 3 años los transistores y procesadores evolucionarían progresivamente; en la actualidad esto se refleja en la evolución de la web 3.0, que hoy en día vincula inteligencia artificial para usos cotidianos y sociales. Sin embargo, estos desarrollos no deben ser interpretados desde el reduccionismo tecnológico, ya que, como menciona Stielger (1998), las tecnologías deben pasar de ser interpretadas como ayudas de la memoria, a ser consideradas como memorias en sí mismas y esto conlleva un reto aún mayor para comprender su función educativa. Por otro lado, los usos de la tecnología en la sociedad también presentan retos asociados a la evolución de la moral, la ética y los medios. Sobre esta última afirmación, Carr (2010) menciona que "la forma en que la web ha ido evolucionando como medio de comunicación reproduce con velocidad cinematográfica toda la historia de los medios de comunicación modernos" (p. 107).

En complemento, la incidencia de las Tecnologías para la Información y la Comunicación (TIC) en la educación ha permitido ampliar la reflexión en torno a la función y rol docente; los modelos pedagógicos han interpretado el uso de la tecnología como una posibilidad para mecanizar la práctica educativa; el conductismo de Skinner (1953) permitió abordar a la conducta como un mecanismo de control a partir de refuerzos positivos y negativos que direccionan el comportamiento, y posicionó al condicionamiento operante como uno de los principales avances de la época. Sin embargo, aunque se reconoce la importancia de este modelo sobre el proceso de enseñanza y aprendizaje, poco se recuerda su postura por incluir tecnología en el aprendizaje, la denominada educación programada (1958) concebía que la organización lógica y secuencial de contenidos permitía generar procesos de autoaprendizaje distanciando la mediación humana. Skinner (2000) en Walden Dos retrata esta noción al concebir la incidencia de la tecnología en el proceso educativo como una posibilidad para agilizar y mecanizar la acción educativa "Lo que está surgiendo en este crítico 
momento de evolución de la sociedad es una tecnología conductista cultural basada únicamente en el refuerzo positivo" (p.273).

El modelo conductista permitió reflexionar sobre el rol de la tecnología en la educación y la función docente como una variable probable de suprimir; la intención de sentido frente a los usos de la tecnología en este contexto hacía énfasis en un proceso instrumental al concebir a la tecnología como herramienta de enseñanza. Esta idea ha tomado fuerza con el paso del tiempo y la evolución tecnológica, Gandara (2012) reafirma lo anterior al mencionar el desarrollo de sistemas de aprendizaje asistido "CAT (Computer Aided Training) ... vieron en la computadora una manera de ofrecer instrucción sistematizada, predecible y reproducible de manera consciente, infinitamente paciente y capaz de dar retroalimentación oportuna al aprendiz" (p. 97). Los primeros intentos de automatizar la práctica docente evidencian una tendencia por desconocer implicaciones pedagógicas, didácticas y comunicativas necesarias para generar aprendizajes significativos; en la actualidad convergen con mayor fuerza discursos que favorecen la irrupción tecnológica en la cultura escolar a través del uso de plataformas educativas que incluyen algoritmos de inteligencia artificial (Claned, Cerego), MOOC, Comunidades Virtuales de Aprendizaje, tendencias educativas con TIC, desde el nivel cognitivo dispositivos de realidad virtual inmersiva o semi-inmersiva que tienen como objetivo generar una sensación de realidad, hasta dispositivos para monitorear procesos cognitivos propios de la atención, memoria y demás procesos ligados a la cognición.

Finalmente, el avance tecnológico también ha permitido ampliar la mirada frente al uso de la tecnología en la educación; la regulación y masificación de la tecnología facilitan la adopción de políticas públicas para concebir los usos tecnológicos más allá de lo instrumental. Los lineamientos de la OCDE (2002) y UNESCO (2003) propenden por la adopción, adaptación y apropiación de tecnologías por parte de la comunidad educativa, esto con el objetivo de mejorar la calidad educativa y potenciar la cualificación docente como medio para trabajar la interdisciplinariedad y el aprendizaje basados en competencias STEM (Ciencia, Tecnología, Ingeniería y Matemática, por sus siglas en inglés) e indicadores de pruebas estandarizadas como PISA. Sin embargo, esta idea de calidad educativa representa solo una visión del uso de la tecnología que ha centrado la mirada en la mediatización de las TIC y que desconoce las características políticas, mediáticas, económicas y educativas de cada región. Una mirada en contexto permite entrever los bajos impactos educativos que ha tenido esta visión, pues como indica el Banco Interamericano de Desarrollo (2012), "la bibliografía disponible documenta que allí donde las tecnologías simplemente han sido incorporadas como nuevas herramientas para hacer lo mismo de siempre, los impactos educativos son escasos o nulos" (p. 252). Estos resultados permiten reflexionar sobre las implicaciones pedagógicas del artefacto tecnológico en la práctica docente como un fenómeno que trasciende las políticas públicas ligadas a reducir la brecha de acceso y que debe ser concebida desde la intención de sentido pedagógico que adquiere del contexto al permitir flexibilizar el proceso de enseñanza y aprendizaje en un sistema educativo que avanza lentamente en comparación con la convergencia mediática.

La tecnología como herramienta no debe ser desprovista de las motivaciones políticas y económicas que orientan su sentido de incorporación en la educación; concebir la tecnología como artefacto cultural implica reconocer los alcances pedagógicos y simbólicos presentes en una sociedad cada vez mayormente interconectada. En consecuencia, la mediación tecnológica debe reflexionar frente a la intención de sentido que adquiere el artefacto en el proceso de enseñanza y aprendizaje como una manera de transformar la práctica docente.

\section{LA INTENCIÓN DE SENTIDO DE LA MEDIACIÓN TECNOLÓGICA EN LA ESCUELA}

La noción de mediación requiere una mirada interdisciplinar, en este orden, se debe observar la configuración de la escuela como institución social que comprende la práctica pedagógica como un ejercicio que adquiere significado en relación con los actores de la comunidad educativa, en esta interacción los modelos pedagógicos 
se han alimentado de saberes de la comunicación y la psicología como una posibilidad para comprender el fenómeno de enseñar y aprender; estos modelos también han evolucionado para incorporar teorías de aprendizaje propias de contextos digitales.

La relevancia que han adquirido las Tecnologías de la Información y la Comunicación (TIC) en las últimas décadas, en particular con los beneficios del trabajo colaborativo de la web 2.0, ha permitido comprender su incidencia en el desarrollo social como su impacto en las dimensiones políticas, económicas, culturales y educativas del ser humano. Al centrar la mirada sobre la educación, se encuentra amplia literatura que aborda las posibilidades de utilizar herramientas tecnológicas para mediar los procesos de enseñanza y aprendizaje en los niveles básicos, medios y superiores (Martin-Barbero, 2009; Silverstone, 1989; Couldry, 2008). La educomunicación como campo de conocimiento amplió la mirada sobre aspectos dialógicos propios de una cultura digital, pero ¿cómo entender el fenómeno de la enseñanza y el aprendizaje en la era de la información y el conocimiento? El conectivismo propuesto por Siemens (2004) presenta una visión que permite comprender la incidencia de los cambios tecnológicos en la actualidad para incorporar los usos de artefactos culturales en la enseñanza y el aprendizaje.

Esta teoría basa sus principios en las posibilidades auto organizativas de la red en donde las personas representan nodos de información que intercomunican ideas, imágenes, símbolos, conceptos a partir de la interacción del conocimiento y el refuerzo de la conexión entre personas (nodos). La autoorganización de la información es un elemento fundamental para filtrar la información presentada de manera azarosa en la web para definir estructuras organizadas útiles al contexto (Rocha, 1998). En el escenario educativo, el conectivismo fomenta la mediación de tecnologías a partir de incorporar recursos, medios, redes sociales y colaborativas a la práctica docente, esto implica que los espacios, tiempos y metodologías que derivan del quehacer docente adquieran una intención pedagógica que otorga sentido a partir de la mediación del artefacto. Siemens (2004) plantea algunos principios para el desarrollo del conectivismo:

1. El aprendizaje y el conocimiento dependen de la diversidad de opiniones.

2. El aprendizaje es un proceso de conectar nodos o fuentes de información especializadas.

3. El aprendizaje puede residir en dispositivos no humanos.

4. La capacidad de saber más es más crítica que aquello que se sabe en un momento dado.

5. La alimentación y mantenimiento de las conexiones es necesaria para facilitar el aprendizaje continuo.

6. La actualización (conocimiento preciso y actual) es la intención de todas las actividades conectivistas de aprendizaje.

7. La toma de decisiones es, en sí misma, un proceso de aprendizaje. El acto de escoger qué aprender y el significado de la información que se recibe, es visto a través del lente de una realidad cambiante. Una decisión correcta hoy, puede estar equivocada mañana debido a alteraciones en el entorno informativo que afecta la decisión.

En consecuencia, la aplicación de los anteriores principios posibilita constituir redes de aprendizaje acorde con los intereses y prácticas sociales propias de las generaciones digitales; los contextos análogos y digitales se presentan como escenarios para la mediación educativa, esta concepción del proceso de enseñanza y aprendizaje resignifica la noción instrumental de la tecnología al generar procesos co creativos para el desarrollo curricular de los contenidos.

En complemento, es necesario reconocer los retos que tiene el modelo educativo por incorporar de manera efectiva la mediación tecnológica de la enseñanza para que los usos generados por la esta no se conviertan en mecanismos instrumentales para la práctica docente (mediatización); Narváez (2019) afirma que "la digitalización electrónica es una mediatización (tecnología), no una mediación (cultura). Asumir la tecnología (generada), el hacer (skills), sin asumir la cultura científica (generadora), el saber, el pensar, el conocer, es lo que nos reproduce como periféricos" (p.14). En otras palabras, si bien la mediatización se apoya 
de recursos digitales, medios y redes sociales, las dinámicas presentes en la relación docente-estudiante no permiten modificar los roles preestablecidos, y en consecuencia mantienen relaciones de poder asimétricas que impiden que los procesos colaborativos se constituyan en redes de aprendizaje desprovistas de los principios referidos por el conectivismo. En igual sentido, la mediación implica un proceso de transmisión de la cultura que se apoya en medios, recursos y redes sociales para trabajar el lenguaje a través de la convergencia de formatos digitales, elementos narrativos, visuales o audiovisuales que transmitan de manera simbólica representaciones, significados y sentidos propios de una cultura digital (Runge, 2012).

Deslocalizar el proceso de enseñanza y aprendizaje como un lugar, permite que los escenarios análogos y digitales converjan para la aplicación de los principios conectivistas, "el aprendizaje es un proceso de conectar nodos o fuentes de información” (Siemens, 2004, p.15). Este proceso requiere integrar las características escritas, orales y audiovisuales presentes en los medios para la transición efectiva de conocimiento. Para Martín-Barbero (2009), se debe pasar de concebir a las tecnologías como ayudas didácticas a concebirlas como una mediación cultural y esto implica una transformación del modelo educativo y en consecuencia de la relación docente estudiante.

Lo que no cabe en la escuela son los nuevos modos de leer y de escribir, que no quieren aceptar ni las Facultades, ni los Ministerios de Educación. Pues se sigue creyendo que lo único que se leen son libros, aunque hoy los adolescentes lean muchos cómics, videojuegos y rock, que son sus nuevas formas de escribir. Pero nuestro anacrónico sistema escolar que cree que la solución es meter la escuela en la televisión en lugar de meter la televisión en la escuela para que los alumnos aprendan a leer las trampas de los noticieros de televisión, la mediocridad de las telenovelas, la estupidez de montones de magazines y sepan apreciar lo poco que haya de valioso en la televisión (p.28).

La perspectiva cultural de la mediación pedagógica se presenta como una oportunidad para transformar la relación docente-estudiante a partir de vincular prácticas sociales y educativas que fomenten la creación de redes de aprendizaje bajo principios conectivistas, lo cual implica reconocer que algunas herramientas, tendencias educativas y lenguajes audiovisuales deben ser concebidos como oportunidades para transformar la escuela al permitir que la práctica docente genere una acción educativa emancipadora que trascienda el uso instrumental de la tecnología.

\section{CONSIDERACIONES FINALES: HACIA OTRAS MEDIACIONES EN LA ENSEÑANZA}

La intención de sentido que se le otorga a la tecnología como mediación de la enseñanza está asociada a múltiples dimensiones que parten por concebir la función de la escuela como institución social que legitima prácticas y saberes propios de la comunidad educativa. En este sentido, incorporar connotaciones políticas y pedagógicas a los artefactos tecnológicos utilizados para mediar el proceso de enseñanza y aprendizaje le permiten a las y los docentes expandir la acción educativa, al vincular fenómenos culturales susceptibles de ser analizados desde las prácticas y saberes propios de una cultura escolar digital. Sin embargo, la experiencia reciente evidencia resistencia al cambio, debido a aspectos propios de una estructura educativa jerárquica, con alta carga administrativa y frecuentes espacios de cualificación sobre lineamientos, decretos y políticas que afectan la gestión educativa, pero que no dialogan en torno a las implicaciones pedagógicas de los artefactos tecnológicos. La flexibilidad curricular y el impacto de discursos económicos frente al cupo escolar, indicadores de calidad educativa y ranking, alimentan una lectura superficial de la cultura escolar digital estática que no conecta con el cambio generacional.

Lo anterior conlleva reconocer significados y narrativas propias de una generación que configura subjetividades y prácticas sociales ligadas a las posibilidades colaborativas y co-creativas que en varios aspectos desbordan concepciones tradicionales de construir conocimiento en la sociedad; la emergencia de las licencias Creative Commons son un ejemplo de cómo la divulgación del conocimiento se adapta a dinámicas de una red del código abierto. Otro ejemplo se evidencia en las tendencias educativas con uso de tecnología, la incidencia del Flipped learning en modalidades e-learning y b-learning flexibilizan la práctica docente al 
tiempo que incorpora intensiones pedagógicas en los recursos digitales utilizados (Ligarretto y Hernández, 2020). Estos y otros debates, propios de una convergencia digital, no se deben distanciar de la escuela, sino, por el contrario, se deben incorporar como fenómenos para la reflexión educativa que generen en las y los estudiantes formas de observarse como agentes activos en la producción de sentido de una tecnología de época.

Reflexionar frente a lo anterior permite desenfocar los discursos educativos sobre la reducción de brechas digitales e indicadores de acceso para enfocar la mirada en narrativas pedagógicas que visibilizan la intención de sentido de la mediación tecnológica en la escuela como un proceso de apropiación cultural. Sin embargo, en este proceso se deben reconocer los beneficios sociales de políticas educativas integrales en Latinoamérica, CEPAL (2010) indica al respecto que "el aprendizaje de estas destrezas ha sido un importante componente de equidad de las políticas de TIC en educación sobre todo en países en desarrollo donde el acceso a las TIC en el hogar es todavía limitado" (p.12). La reducción en las brechas digitales, como los procesos de alfabetización digital, presentan un primer paso para transformar la escuela; programas como Computadores para Educar (Colombia), PROUCA (Brasil), Ceibal (Uruguay, Perú) visibilizan estrategias educativas para fomentar el desarrollo de competencias TIC en los maestros y maestras. Sin embargo, la velocidad de evolución tecnológica propende por una sociedad con mayor interconexión y dependencia tecnológica, este hecho debe expandir los programas actuales en políticas educativas sostenibles donde el orden cualitativo de la apropiación tecnológica desborde los datos cuantitativos de acceso y generen comunidades educativas propias de una cultura digital. Asimismo, el proyecto Neuralink plantea un futuro de simbiosis digital y cognitiva que deben abrir nuevos horizontes de análisis para la educación, de igual forma, el alcance de la escuela, como institución social que conserva y reproduce la cultura, debe asumir una postura crítica a la luz de fenómenos como fakenews, sexting, cyberbullying, entre otras prácticas sociales que fomentan ejercicios de parametrización y control del consumo digital en todos los sectores etarios, pero con especial preocupación, en el proceso educomunicativo como una manera de aprender a leer una nueva realidad que desborda las fronteras análogas y digitales al centrar el debate sobre las implicaciones éticas de los usos tecnológicos en la escuela.

Por otro lado, una mirada reflexiva sobre las relaciones históricas de la tecnología y la educación denotan una tendencia por la automatización de algunas funciones del maestro. El sistema CAT y un modelo conductual de época generaron los primeros intentos por reemplazar el rol de los maestros y maestras, aunque resulta evidente que los desafíos planteados por la evolución tecnológica, que vinculan realidad aumentada e inteligencia artificial, plantean nuevos desafíos para la práctica docente las cuales deben adaptarse a una cultura de lo digital. En este sentido, el movimiento Edupunk desarrollado por Piscitelli (2012), busca transformar las prácticas educativas dentro y fuera de la escuela para reconfigurar el imaginario de la tecnología como una posibilidad co-creativa para el aprendizaje. Algunos de los resultados de la experiencia desarrollados por Arbor (2011) enfatizan en una pedagogía para desbordar los márgenes institucionales que, a partir de procesos colaborativos, en particular de Wikis, fomentan una acción activa de las y los estudiantes.

A mi juicio, esta experiencia educativa, junto con otras mencionadas en el texto, evidencian una posibilidad de expandir el triángulo pedagógico (maestros y maestras, contenido y estudiantes) al incorporar dimensiones culturales específicas de la mediación tecnología. La escuela actual necesita fomentar principios conectivistas que trasciendan el aula y establezcan nuevas relaciones entre la comunidad educativa, los padres de familia como agentes activos de la acción educativa, al igual que maestros y maestras que medien artefactos tecnológicos desde lo pedagógico y comunicativo para interconectar ideas y generar redes de aprendizaje entre la comunidad educativa; el carácter cultural de la acción educativa comprende una dimensión instrumental de la herramienta, pero enfatiza en el significado social de características contextuales y prácticas que giran en torno a la apropiación de la tecnología en las generaciones digitales.

Lo anterior, conlleva incorporar nuevos formatos audiovisuales, redes sociales, memes, lenguaje visual, y todo un ecosistema digital que, desde hace un tiempo atrás, media nuestras relaciones laborales, familiares 
y sociales. El acceso y disposición de la información representa el primer recurso para generar disposición al aprendizaje que debe ir acompañado por habilidades y competencias docentes que propendan por reforzar la capacidad de interconexión a partir del trabajo colaborativo. Este reto resulta aún mayor en maestros y maestras que conciben una relación circunstancial con la tecnología, internet como nuevo medio que busca reemplazar a la radio y la televisión; sin embargo, lo mencionado anteriormente frente a las dimensiones políticas, económicas y la evolución misma de la web obliga a reflexionar frente a cómo el artefacto cultural transforma dinámicas propias de lo educativo. La mediación digital de la enseñanza no refiere a conocer las herramientas y aplicativos, sino, por el contrario, a reconocer una relación histórica (Techne) y asumir a la tecnología en sí misma como una manera de comprender una cultura de lo digital, en donde la pregunta educativa no indaga por el qué sino por el cómo y el porqué de la mediación tecnológica en la enseñanza. De lo contrario, la brecha por comprender las prácticas y saberes de lo digital distanciara con mayor fuerza a las docentes y estudiantes que observan con ojos distintos acceder, utilizar, consumir y crear con tecnología; una cultura escolar digital necesita incorporar formatos y narrativas digitales que desborden lógicas propias del pensamiento científico, la oralidad del podcast, el lenguaje visual del meme, la multimodalidad de las redes sociales, los procesos co-creativos transmediales. Entre otras prácticas de internet establecen nuevas maneras de crear y divulgar conocimiento educativo por fuera del canon tradicional del lenguaje escrito. Esta oportunidad de transformar la escuela debe convocar una acción conjunta por parte de los maestros y maestras y demás agentes de cambio para adaptar contenidos y estrategias didácticas al escenario de la mediación digital, en donde se propenda por un uso reflexivo del artefacto tecnológico analizado desde narrativas propias del consumo digital de los y las estudiantes, pues de lo contrario, la lenta transformación de la escuela distanciará los aspectos dialógicos, emocionales y comunicativos que debe concebir una práctica educativa propia de la era de información y el conocimiento.

Hoy más que nunca se requiere generar un sentido crítico frente a la incorporación de la tecnología en la escuela, pues la exposición constante a la información, la falta de criterios para validar fuentes y el consumo mediático basado en entretenimiento, reivindica la importancia de la labor de las y los docentes en la era digital, que a diferencia de los intentos anteriores por automatizar la acción educativa presentan una era de la información mediada por artefactos tecnológicos que reivindican la pertinencia pedagógica de las y los docentes como agentes de transformación para fomentar una lectura crítica de los cambios que le impone los estándares educativos de la digitalización. Ahora bien, este proceso de transformación requiere reconocer la velocidad tecnológica para incidir en las prácticas sociales con el uso de tecnología; la coyuntura actual evidencia desigualdades educativas históricas, en particular, en el contexto latinoamericano inician por la falta de conectividad, la poca inversión pública, el lento relevo generacional y una mirada educativa carente de las conexiones entre el diseño y la comunicación como agentes que dinamizan las prácticas educativas de una escuela moderna en constante transformación, un ejemplo de ello se evidencia en el modelo educativo Finlandés en donde los diseños de Rosan Boch reflejan una concepción integral de la función de la escuela al generar espacios para la práctica reflexiva de los maestros y maestras.

Una política educativa integral para incorporar tecnología en la escuela debe deslocalizar el aprendizaje como lugar y requiere concebir los procesos de enseñanza y aprendizaje como escenarios que trasciende los aspectos analógicos de la práctica educativa tradicional, al reconocer que las dinámicas sociales de generaciones atravesadas por el uso de tecnología permiten establecer una cultura digital propia de la escuela actual. La mediación pedagógica de la tecnología reconoce cómo dimensiones comunicativas apoyan con mayor frecuencia la acción educativa, este hecho posibilita un campo de experimentación para las maestras y maestros que conciben una mediación tecnológica que incorporan las prácticas y saberes de una cultura digital.

¿Cómo potenciar la mediación tecnológica para flexibilizar la práctica docente?, ¿El aprendizaje en red implica deslocalizar el aprendizaje como lugar?, ¿cómo vincular fenómenos mediáticos digitales al currículo?, ¿por qué analizar por separado dinámicas análogas y digitales? Estas y otras preguntas permiten puntos de 
encuentro entre una generación digital y una educación que piensa otras formas de mediar la enseñanza en tiempos donde la tecnología conserva, reproduce y transforma los fenómenos educativos de una sociedad contemporánea mediada por la tecnología. Esto implica recordar al final que todo acto educativo es un acto comunicativo en sí mismo (Kaplun, 2002) y esto conlleva trascender barreras análogas y digitales para pensar en la escuela como un nodo que interconecta y fomenta redes colaborativas entre una comunidad educativa que utiliza, consume, crea y media con tecnología.

\section{REFERENCIAS BIBLIOGRÁFICAS}

Arbor. (2011). ¿Es factible el Edupunk en la formación universitaria española? herramientas 2.0, confeccionando espacios de formación. Revista Ciencia, Pensamiento y Cultura, 187, 213-217. doi: https://doi.org/10.3989/ar bor.2011.Extra-3n3147

Banco Interamericano de Desarrollo [BID] (2012).Educación para la transformación. Biblioteca Felipe Herrera del Banco Interamericano de Desarrollo. Madrid, España: Siglo XXI editores.

Borges, J.L. (1996). Las Cosas. México: Emecé Editores.

Cairncross, F. (1997). The Death of Distance. Londres: Orion Ediciones.

Carr, N. (2010). Superficiales ¿Qué está haciendo internet con nuestras mentes? Buenos Aires, Argentina: Editorial Santillana.

Castells, M. (1999). La era de la información: economia, sociedad y cultura. La sociedad red. Madrid, España: Siglo XXI editores.

CEPAL (2010). Impacto de las TIC en los aprendizajes de los estudiantes. Naciones Unidas. Santiago de Chile: Siglo XXI editores

Couldry, Nick (2008). Mediatization or mediation? Alternative understandings of the emergent space of digital storytelling. New media \& society, 10(3), 373-391.

Elbaz, M y Helly, D. (1996). Modernidad y postmodernidad de las identidades nacionales. Revista internacional de filosofia politica, 10(7). 124-137. Recuperado de https://dialnet.unirioja.es/servlet/articulo?codigo $=2704743$

Esposito, R. (2016). Las personas y las cosas, Katz. Buenos Aires: Planeta Ediciones.

Foucault, M. (1968). Las palabras y las cosas. Una arqueología de las ciencias humanas. México: Siglo XXI.

Gandara, M. (2012). Las tecnologias de la información y la comunicación: Una introducción para educadores. España: Editores Océano.

Kaplun, M. (2002). Una pedagogía de la comunicación. La Habana: Editorial Caminos.

Ligarretto, R, y Hernández, H. (2020). Evaluar el modelo Flipped learning en un Ambiente de Aprendizaje E-learning. Revista metavaliacao, 12(36), 573-600. doi: http://dx.doi.org/10.22347/2175-2753v12i36.2575

Martín-Barbero,J. (2009). Cuando la tecnología deja de ser una ayuda didáctica para convertirse en mediación cultural. Teoría de la Educación. Educación y Cultura en la Sociedad de la Información, 10(1),19-31. Recuperado de http s://revistas.usal.es/index.php/eks/article/view/15549

Moore, G. (1975). Progress in digital integrated electronics. IEEE International Electron Devices Meeting, 10(8),11-13. Recuperado de https://www.eng.auburn.edu/ agrawvd/COURSE/E7770_Spr07/READ/Gordon_Moore_1 975_Speech.pdf

Narváez, A. (2019). Comunicación educativa, educomunicación y educación mediática: una propuesta de investigación y formación desde un enfoque culturalista. Palabra Clave, 22(3), 22-31. doi: http://doi.org/10.5 294/pacla.2019.22.3.11

Organización de Estados Iberoamericanos para la educación, la ciencia y la cultura [OEI]. (2001). Ciencia, Tecnología y Sociedad: una aproximación conceptual. Cuadernos de Iberoamérica. Santiago de Chile: Siglo XXI editores.

Organización para la Cooperación y el Desarrollo Económico [OCDE]. (2002). The Definition and Selection of key Competencies. Executive Summary. Estados Unidos: Siglo XXI editores. 
Piscitelli, A. (2012). Edupunk aplicado aprender para emprender. Fundación Telefónica. España: Editorial Ariel. Barcelona.

Rocha, L. M. (1998). Selected Self-Organization and the Semiotics of Evolutionary Systems. Revista Indiana, (32). Recuperado de https://link.springer.com/chapter/10.1007/978-94-017-1510-2_25

Runge, A. K. (2012). El pensamiento pedagógico y didáctico de Juan Amós Comenio: su papel en la pansofía triádica. Pedagogía y Saberes, (36), 93 -107. doi: https://doi.org/10.17227/01212494.36pys93.107

Siemens, G. (2004) Learning and Knowing in Networks: Changing roles for Educators and Designers. Culture and TIC, (18). Recuperado de https://eclass.uoa.gr/modules/document/file.php/PPP233/\%CE\%AC\%CF\%81\%C E\%B8\%CF\%81\%CE\%B1\%20\%CE\%B2\%CE\%B9\%CE\%B2\%CE\%BB\%CE\%B9\%CE\%BF\%CE\%B3\%CF\%8 1\%CE\%B1\%CF\%86\%CE\%AF\%CE\%B1\%CF\%82/Siemens\%202008.pdf

Silverstone, R. (1989). Let us then Return to the Murmuring of Everyday Practices: A Note on Michel de Certeau, Television and Everyday Life. Theory, Culture and Society, 6(1), 77-94. Recuperado de https://journals.sagepu b.com/doi/10.1177/026327689006001004

Skinner, B.F. (1953). Science and human behavior. [ Ciencia y comportamiento humano]. New York: Macmillan Editors.

Skinner, B.F. (2000). Walden dos. Bogotá, Colombia: Editorial Planeta.

Smith, A. (1983). Theories of Nationalism. Londres: Cambridge University Press, MacMilIan Editors.

Stiegler, B. (1998). Technics and Time 1: The Fault of Epimetheus. California, Estados Unidos: Stanford University Press.

United Nations Educational, Scientific and Cultural Organization (UNESCO), (2003a). Las tecnologias de la información y la comunicación en la formación docente: guia de planificación. España: UNESCO Ediciones. Recuperado de https://bit.ly/1BG1Rv7

Winner, L. (1985). Do Artifacts Have Politics?. Philadelphia, Estados Unidos: American Academy of Arts and Science. Recuperado de https://www.cc.gatech.edu/ beki/cs4001/Winner.pdf

\section{INFORMACIÓN ADICIONAL}

Cómo citar: Ligarretto Feo, R.E. (2021). Mediación tecnológica de la enseñanza: Entre artefactos, modelos y rol docente. Revista Educación, 45(2). Recuperado de http://doi.org/10.15517/revedu.v45i1.42999 